\title{
Nuevas tendencias en el estudio del Sindrome Diarreico en la niñez
}

\author{
DRES.: HLMBFRTO A. SORIANO *** y BUFORD L.. NICHOLS *
}

EJ Sindrome Diarreico es uno de los problemas no resueltos más antiguos del hombre. La complejidad enorme de esta afección se entiende con facilidad si se considera que afecta a un sistema como el gastrointestinal cuya función es transformar los alimentos en una solución acuosa que pueda circular en la sangre sin dañar el organismo que a la vez contiene un gran número de substancias nutritivas molecularmente muy diferentes que deben ser transportadas atravesando, al menos, dos barreras celulares y muchas veces en contra de una gradiente electroquímica. (1-5) Para rcalizar esta función el aparato digestivo cuenta con una morfología complicada que tiene una superficic real comparable a la de una cancha de tenis, con un recambio celular tal que la superficie entera es reemplazada cada 3 a 4 días, (20 a $50 \mathrm{mi}-$ llones de células por minuto), y con una diferenciación celular de gran especialización. (6-10).

Este sistema está abierto al medio externo y contiene en su interior una población microbiana polimorfa, metabólicamente activa y con una capacidad de reproducción que, de no ser contenida por los mecanismos de control de aparato digestivo, una sola de las especies que habitan el intestino sería capaz de desarrollarse en 24 horas hasta ser una masa metabólicamente equivalente a la de un niño de dos años. $(11-14)$. El aparato digestivo no sólo debe mantener el control sobre la cantidad de gérmenes que lo habitan sino también debe regular la calidad de la flora microbiana, desarrollando procesos capaces de eliminar selectivamente las bacterias inútiles o dañinas. (15-17). Entre otros mecanismos, la mucosa intestinal cuenta con

\footnotetext{
- Sección de Nutrición y Gastroentcrología, Departamento de Pediatría, Escuela de Medicina de Baylor y Hospital de Niños de Texas, Houston, Texas. U. S. A

* Departamento de Pediatría, Facultad de Medicina de la Universidad de Chile, Area Norte. Hospilal Roberto del Río.
}

300.000 células por milímetro cúbico en su lámina propia capaces de producir inmuno globulina A secretora cuya función, no bien entendida aún, sería la de defender el organismo de la invasión de antígenos potencialmente dañinos para el organismo. (18-22). Por último, el aparato digestivo debc enfrentar con éxito el desafío de una carga de alimentos provenientes del exterior de cantidad, composición y contaminación muy variadas, mezclarlas con sus productos de secreción hasta un volumen promedio aproximado de 10 litros diarios, extraer las substancias nutritivas, esterilizar la solución que finalmente alcanza la sangre y eliminar una mezcla concentrada de desechos que habitualmente no sobrepasa los 200 gramos diarios. De esta eficiencia depende esencialmente la capacidad del ser humano para mantener su supervivencia, su estado nutritivo y su salud. $(4,23-26)$.

En síntesis, se puede considerar el aparato digestivo humano como un ecosistema de equilibrio inestable compuesto por un grupo de órganos de compleja estructura y elevada especialización, con mecanismos de control, algunos dependientes de otros sistemas, con una microflora abundante y variada y con una carga continua de substancias y microorganismos provenientes del medio exterior. De este ecosistema depende principalmente la salud de la persona.

EPIDEMTOLogía-- Una síntesis muy breve, pero no por eso menos trágica, de lo que ocurre en los países menos desarrollados es que nacen muchos niños que comen poco, tienen muchos episodios diarreicos, se desnutren, se deshidratan y se mueren en una alta proporción, la mayoría de ellos en el hospital. Los que sobreviven Ilegan, con secuelas, a ser adultos (27-33). 
No cabe duda que la desnutrición y el Sindrome Diarreico (SD) forman un círculo vicioso de causa - efecto, que puede iniciarse con cualesquicra de las dos. (23, 34-38). Sin considerar aspectos éticos mucho más profundos, es necesario destacar el elevado costo que significa para una nación pobre cl tratamiento del Sindrome Diarreico y la Desnutrición Infantil. (39-43).

Sin embergo, no sólo para las naciones pobres es un gran problema; también es uno de los riesgos do exfermar más importantes para la población de naíses industrializados. (44-51). Es una de las causas más frecuento de ausentismo cn el trabaio y es uno de los riesgos más comunes para grupos institucionales (fuerzas armadas, colegios, etc.), para grupos minoritorios con haio nivel de vida y para las persorns que viajan. (5?-55). En estos países se ha logrado disminuir sustancialmente el riesgo de morir pcro no en la misma medida cl riesgo de enfermar. (56-58).

Los cstudios epidemiológricos han señalado persistente y consistentemente al SD en general como una entidad con caracteristicas epidemiológicas propias de una enfermedad infecciosa, csto es, con agente, hućsned, ambiente y mecanismos de transmisión con eventuales períodos epidémicos sobre una base endémica o hiperendémica $(17,27,33,44$, 59-61). Sin embargo, cerca del $80 \%$ de los casos no tienen un agente demostrable, (62. 65) la reacción de diferentes hióspedes puede ser complctamente diferente. $(66-70)$ la influencia de algunos factores ambientales puede ser muy variable de una población a otra (71-74) y los mecanismos de transmisión, con algumas excepciones, no han sido claramente demostrados. Esto, a nuestro iuirio, señala que existen muchos grunos de "cnfermedades diarreicas" que tienen en común las características de enfermedad infecciosa pero con epidemiologías diferentes.

Como medidas preventivas del SD se ha puesto mucho énfasis en el saneamiento del ambiente, la mejor educación biológica y el aumento de la frecuencia y duración de la lactancia natural. $(23,27,34)$. A nuestro juicio los dos primeras medidas revelan en cierta manera la falta de conocimientos suficientes sobre el SD, porcue son medidas de carácter tan general que podrían resolver cualesquicra de los problemas de salud de las naciones pobres e ignorantes. Pero son do elevadísimo costo y no reflejan la aplicación de un conociniento específico sobre las caulsas y mecanismos de un problema para resolverlo. Ia icrcera medida, esto es, el fomento de la alimentación natural, también refleja nuestra falta de conocimiento preciso. Sabemos que existe menos riesgo de enfermar para el niño alimentado al pecho $(23,33$, 43 ), pero no sabemos exactamente por qué; sabemos que hav la tendencia universal a la disminución de la frecuencia y duración de la alimentación natural, pero no sabemos exactamente por qué. Por último, por muchos estudios antropológicos, sabemos que modificar esta situación es más difícil y costoso que el sarieamiento y la cducación biológica de una sociedad, porque es un fenómeno que tiene raíces más profundas y relaciones más complejas.

Por lo anteriormente expuesto es necesario concebir, sn futuros trabajos epidemiológicos sobre el Síndrome Diarreico Agudo (SDA) en la niñez, la necesidad de ina relación con la etiolosía específica, la fisiopatología, la clínica y la terapéutica. Creemos que con una aproximación multidisciplinaria y multinacional sería posible establecer dichas relaciones en beneficio de una mayor comprensión de los factores en juego en el SD en la niñez.

Microblologin.- Los estudios microbiológicos han señalado al menos 3 grupos de agentes capaces de provocar el SD: las bacterias. los virus y los parásitos. $(17,27,56$, 63). Históricamonte se han reconocido gérmenes capaces de provocar el SD cumpliendo los postrulados de Koch: Shigellas, Salmonellas, Cólera vibrio, y posteriormente Estafilococo, ECEP, Yersinia enterocolítica y otros. Estos grérmenes actúan invadiendo la mucosa intestinal, producicndo toxinas, o por ambos mecanismos $(1,16,48,75,76)$. Desde largo tiempo también se ha sospechado el posible papel patogénico de algunos otros gérmenes, aue se ha confirmado recientemente con la demostración de cepas de E. coli y Klebsiella capaces de acción patógena a través de toxinas. $(11-48,61,74,76)$. Otros gérmenes, también según publicaciones recientes, podrían causar diarrea a través de la modificación del contenido intestinal, produciendo substancias capaces de desencadenar o mantener el cuadro, (ácidos biliares secundarios; hidroxiácidos grasos, ácido láctico, etc.). $(1,3,4,12,17)$. También es posible que muchos gérmenes actúen estimulando o inhibiendo substancias que tienen una acción fisiológica en condiciones normales pero que pueden desencadenar el proceso diarreico al aumentar a disminuir su concentración (AMP cíclico, prostaglandinas, etc.). $(1,3,6,13,77)$. Por otra parte, se ha demostrado que no sólo el tipo de germen tiene 
importancia, también su ubicación y concentración en el tracto digestivo puede tener valor patogénico: La invasión de las partes altas del intestino delgado por flora colónica habitual puede desencadenar el SD. (2, 14, $16,78)$. Esto abre una perspectiva no señalada antes por la epidemiología: la auto infección exógena (ciclo ano-mano-boca en el mismo individuo) y la auto infección endógena (vía colón-yeyuno-ileon-duodeno en el mismo individuo).

El papel etiológico de los virus ha sido demostrado largamente en epidemias relacionadas con diversas especies bien identificadas $(62,63,75)$, lográndose reproducir la enfermedad en voluntarios. Recientemente, se ha podido demostrar la presencia de virus en la mucosa intestinal y heces de pacientes con SD, su ausencia en el grupo control, y las reacciones serológicas específicas correspondientes (79-81). Queda por establecer la frecuencia de estos virus en el grupo de SD, el o los mecanismos directos de acción sobre la célula intestinal y los posibles mecanismos indirectos a través de proliferación bacteriana anormal sobre una mucosa previamente dañada, como ocurre en las infecciones de la mucosa respiratoria.

El papel etiológico de los parásitos en el SD también demostrado desde largo tiempo, no ha sido tema de actualidad en las publicaciones de los últimos años, a pesar del hallazgo de infestaciones intestinales masivas por parásitos en algunos de los grupos estudiados. También es un aspecto que debe ser considerado en el estudio de las causas y mecanismos de enfermedad en el SD en la niñez $(37,71,82)$.

El estudio de la respuesta de la microflora intestinal al tratamiento antibiótico dio origen al descubrimiento de los plásmidos transferibles de resistencia a drogas (83-86). EIlos son partículas extracromosómicas de DNA que pueden ser transferidas entre diferentes especies de un mismo género o entre géneros diferentes de bacterias, entregando, de esta manera, la información genética necesaria para desarrollar la resistencia a una o múltiples drogas $(81,88)$. Tanto o más importante que lo anterior, se ha visto que la capacidad de producir enterotóxinas estaría condicionada por un plásmido, esto es, una partícula transferible capaz de inducir la producción de enterotóxina en el germen receptor (83-88). Una lista de los bacterios que pueden intercambiar plásmidos incluye el género Escherichia, Shigella, Salmonella, Proteus y Klebsiella, entre otros.
FISIOPATOLOGIA

EI SD es la insuficiencia del aparato gastrointestinal para controlar la eliminación fecal de nutrientes, principalmente agua y electrolitos. Esta insuficiencia puede ser debida a un aumento de la secreción gastrointestinal, a una disminución de la absorción o ambas. La causa última es la incapacidad de las células que componen la llamada "Unidad funcional del intestino" (vellosidad, cripta y lámina propia) para transportar nutrientes (agua y sales, esencialmente) desde el lumen intestinal hacia la circulación y/o para controlar el paso de agua y iones desde la circulación hacia el lumen intestinal. El resultado obvio es la tendencia del organismo a la deshidratación, los trastornos del metabolismo electrolítico y la desnutrición. ¿Por qué puede producirse el fracaso de la "Unidad funcional intestinal"?

1) Por supresión (como en extensas infecciones, necrosis o resecciones).

2) Por alteración morfológica microscópica (como en afecciones que se acompañan de daño de las vellosidades con diferentes grados de aplanamiento del epitelio.).

3) Por alteración morfológica ultramicroscópica y/o funcional (como en afecciones producidas por toxinas, otras substancias químicas ingeridas o productos secundarios del metabolismo en el lumen intestinal).

4) Por insuficiente tiempo de contacto de la "Unidad funcional" normal con el contenido del lumen intestinal (como en afecciones que se acompañan de motilidad propulsora aumentada).

5) Por cambios en las características físicoquímicas del contenido intestinal que exceden la capacidad de adaptación de la "Unidad funcional" normal (como en afecciones con succus estéricus de osmolaridad aumentada, o sin adecuada formación micelar, o de volumen exagerado, etc.).

6) Por aumento de la presión hidrostática del sistema de drenaje de nutrientes hacia la circulación (como ocurre en afecciones con ectasis linfático o venoso, etc.).

7) Por la acción simultánea de uno o más de los mecanismos mencionados. 
En el aparato digestivo, cualquiera de los órganos que lo componen puede ser responsable; primaria o secundariamente, del SD: El estómago, como en el Sindrome de Zollinger-Ellison; el hígado, como en las afecciones que se acompañan de inadecuado flujo $\mathrm{y} / \mathrm{o}$ composición de la bilis; el páncreas, como en la fibrosis quística; el intestino delgado, como en ciertas infecciones (por ejemplo, Cólera, E. coli toxigénica, Lamblias, virus) $y$ en ciertas anomalías congénitas (alactasia y otras), etc.; o el intestino grueso como en ciertas infecciones (Shigellosis, Amebiasis, etc.), o en ciertas anomalías congénitas (cloridorea y otras), etc. En el SDA en la niñez, se ha postulado que la causa inicial más frecuente es un agente vivo o sus productos. Esto no ha sido demostrado. Es necesario estudiar la frecuencia de bacterias toxigénicas y nuevos virus como agentes responsables antes de dar una respuesta más definitiva (89-91).

Cualquiera sea el agente infectante, las condiciones del huésped que lo recibe tienen influencia decisiva en la producción de enfermedad, y sus consecuencias. La desnutrición por sí misma provoca alteraciones del aparato gastrointestinal $(38,92)$, la mayoría de las cuales requieren mayores estudios como la disminución del índice mitótico en el niño marásmico, atrofia de vellosidades e infiltración de la lámina propia en el desnutrido pluricarencial, hipomotilidad propulsora o relajación de la musculatura circular, colonización de las partes altas del intestino por flora microbiana en cantidad y calidad anormal, defecto del sistema inmunitario intestinal, etc. La desnutrición, la edad y la alimentación del niño influyen en la composición corporal de agua y sales y la capacidad reguladora del metabolismo hidrosalino en forma tal que frente a una misma situación de pérdida de agua y sales, la célula y los órganos de control no logran actuar con la misma eficacia que en el niño bien nutrido o de mayor edad $(70,93)$. Sin embargo, la mayoría de estos fenómenos, como la respuesta osmótica limitada de la célula, incapacidad celular de retener potasio y mantener bajas concentraciones de sodio, la insuficiencia tubular renal relativa, etc., requieren de mayores estudios para su comprensión y manejo terapéutico (9496).

El o los mecanismos desencadenados por los agentes causales conocidos (que no constituyen más del $20 \%$ de los casos) han sido estudiados extensamente en personas adultas y, más restringidamente, en niños. Sin embargo, las conclusiones están muy le- jos de aclarar los mecanismos, aún de estos agentes causales conocidos: El consenso casi unánime de que el vibrio cólera actúa sin provocar lesiones microscópicas del epitelio intestinal ha sido denegado en recientes publicaciones (97). Por otra parte, se ha visto que otras enterotoxinas pueden provocar alteraciones ultramicroscópicas de las microvcllosidades, del cuerpo celular y de los espacios intercelulares del epitelio intestinal $(98,99)$; los escasos estudios sobre las características de la mucosa intestinal en los casos de SD sin etiología precisada, a través de la biopsia intestinal, han dado resultados más bien contradictorios y en general no han sido relacionados con las alteraciones funcionales (100-103); se ha visto que el aumento del AMP cíclico en la célula intestinal es un factor común para muchos agentes productores de SD y no sólo para los provocados por enterotoxinas; etc.

Por otra parte, con frecuencia se olvida que el agente causal puede ser sólo un mecanismo de gatillo que desencadena procesos que perpetúan el SD. Así, la colonización bacteriana secundaria del intestino por gérmenes "fecales" puede producir metabolitos anormales (ácidos biliares secundarios, etanol, hidroácidos, etc.) que mantengan la lesión después que el agente causal ha sido removido (104); la lesión inicial de las microvellosidades puede conducir a una intolerancia a disacáridos cuyo efecto osmótico en el intestino delgado y su eventual degradación a ácidos orgánicos con inflamación del intestino grueso, puede mantener el SD; también la desnutrición provocada o agravada por el período inicial de SD, puede tener influencia decisiva en su perpetuación (105-106). En todo caso, estos mecanismos secundarios de mantención y agravación del SD deben ser motivo de mayores estudios porque su importancia terapéutica puede ser insospechada.

\section{CLINICA Y TERAPEUTICA}

Los estudios clínicos del SD en la niñez han sido poco alentadores. El estudio de los síntomas y signos clínicos y exámenes de laboratorio ha demostrado que es posible orientarse pero no decidir sobre la etiología en la inmensa mayoría de los casos (107-111). El estudio de las alteraciones de la secreción y absorción de diferentes nutrientes y de las alteraciones del metabolismo hidrosalino no ha entregado herramientas fáciles de aplicar en la práctica coti- 
diana $(104,106,112)$. Es indispensable insistir en el estudio de síntomas y signos de acuerdo con la edad del paciente, con su estado nutritivo y su condición socio-económico-cultural y sanitaria, relacionándolos con la etiología y la fisiopatología. Es necesario buscar nuevos elementos de diagnóstico etiológico como la citología fecal (113), el análisis de contenido intestinal, las pruebas biológicas de patogenicidad, etc. Es también necesario insistir en la correlación de los signos clínicos con los trastornos de la secreción y absorción de nutrientes a través de exámenes que permitan, como en el caso de la malabsorción de hidratos de carbono (105, 114 ), detectar fácilmente las alteraciones en la absorción de hidratos de carbono de proteinas y grasas y otras sustancias que pueden estar condicionando la diarrea (ácidos biliares libres, hidroxiácidos, etc.). Por otra parte, la interpretación de las variaciones de hidratación y electrolitos plasmáticos en el SDA. debe ser revisada críticamente en el niño de diferentes edades, bien nutrido y malnutrido (115). Los signos clínicos de deshidratación, los signos de depleción de sodio y potasio y su diferencia con la pseudodepleción (116), el papel de los trastornos del magnesio en las manifestaciones clínicas etc., deben ser motivo de mayor estudio.

Por último, si bien el manejo terapéutico del SD ha sido motivo de numerosos estudios, aún existe mucho por aclarar. El tratamiento antimicrobiario se ha demostrado parcialmente eficaz en algunos casos e ineficaz en otros (dependiendo de la etiología, de la droga empleada y de los parámetros de observación) (65, 117-120). Existiendo un agente microbiano responsable, es lógico insistir en la búsqueda de nuevos medicamentos con que combatirlo sin olvidar que, muchas veces, puede representar sólo el punto de partida de otros procesos que requieren más de un manejo dietético adecuado o, eventualmente, de otras drogas. El tratamiento antidiarreico "inespecífico", generalmente sobre la base de drogas que alteran la motilidad intestinal, no sólo ha dado resultados contradictorios sino que, en algunos casos su uso podría estar contraindicado (120-121). La acción de las drogas llamadas clásicamente "demulcentes protectores y absorbentes" es un campo abierto a la revisión ya que su o sus mecanismos de acción podrían ser diferentes a los supuestos. En este sentido, los resultados obtenidos con una resina de intercambio iónico, la colestiramina, están siendo activamente revisados (122). El tratamiento dietético ha evo- lucionado desde el uso empírico de leches modificadas en su concentración, acidificación, etc., hasta el empleo de fórmulas modulares que permiten el manejo seleccionado de diferentes fallas de absorción, pasando por las "leches" de diferentes leguminosas, las leches libres de hidratos de carbono, etc. (123-125). El uso clínico de cada una de estas modificaciones aún está restringido en muchos casos a pacientes incluidos en trabajos de investigación. La decisión de usar una dieta accesible, racional y científica, debe ponerse al alcance del clínico, para lo cual aún es necesaria mayor investigación básica y aplicada. Vale la pena, sin embargo señalar dos avances de interés práctico en el manejo dietético de SD de evolución tórpida: 1) El uso de miel de abejas como fuente de fructosa en el intento de administrar carbohidratos en los casos con intolerancia a los disacáridos y a la glucosa $(125) ; 2) \mathrm{EI}$ uso de aceite de coco como fuente de triglicéridos de cadena media en casos con malabsorción de grasas por insuficiente formación micelar. Dichos triglicéridos no requieren la acción de los ácidos biliares para su absorción (125, 128, 129).

El tratamiento de prevención y reparación de los trastornos hidrosalinos tuvo un impresionante avance en los últimos 30 años hasta llegar al manejo sofisticado que se realiza en los centros médicos académicos de la mayoría de los países desarrollados: Control casi permanente de los valores sanguíneos y de la función renal, uso de técnicas de infusión continua seguras y prolongadas, etc. $(126,127)$. En este sentido, no cabe duda que, si la alimentación total parenteral estuviera exenta de los riesgos que tiene, sería la medida terapéutica ideal en casos de SD crónico inespecífico, en los cuales el mejor tratamiento sigue siendo el "reposo gastrointestinal" que se obtiene con una dieta cero por boca, mantenida por períodos prolongados de tiempo (semanas a meses) $(126,127$, 130). Sin embargo, la necesidad de un estricto control médico, clínico y de laboratorio y de enfermería para evitar las complicaciones metabólicas e infecciosas $(131,132)$ de la alimentación total parenteral, hacen que la situación sea distinta en lugares que no disponen de centros médicos académicos como en los países industrializados, que es la condición de la mayoría de los países en desarrollo. Es distinta no sólo por la falta de recursos para un tratamiento sofisticado, sino también porque los pacientes son diferentes. Aún queda mucho por definir sobre cuánta y qué solución a soluciones deben ser 
empleadas en niños de diferente edad, estado nutritivo, en niños que están recibiendo alimentación materna, etc. $(27,69,93,133)$. Aún queda mucho por conocer sobre el papel de otros elementos (magnesio, elementos trazas, fosfatos, etc.) en el tratamiento y prevención de la deshidratación y los trastornos electrolíticos del niño con SDA (112, 115, 116).

Tanto el uso de dietas especiales por vía oral como el tratamiento sobre la base de agua, sales y otros nutrientes por vía parenteral deben interpretarse como tendientes a los siguientes fines:

1. Prevenir o corregir trastornos agudos de la nutrición que pueden causar la muerte como son la deshidratación, los trastornos electrolíticos específicos y del equilibrio ácido base.

2. Prevenir o corregir trastornos crónicos de la nutrición secundarios o concomitantes, en todo caso agravantes, del proceso diarreico, tales como la desnutrición marásmica o próieica, déficit vitamínico o electrolítico, etc.

3. Facilitar la recuperación de la alteración morfológica y funcional del aparato gastrointestinal responsable del proceso diarreico.

\section{REFERENCIAS}

1.-Low-Beer, T.S. and Read, A.E.: Progress Report Diarrhea: Mechanism and Treatment. Gut 12: 1021-1036, 1971.

2.-McCarthy, C., Love, A.H.G., Low-Beer, T.S.: Modern Concepts in the Aetiology and Management of Diahrroea. Irish J. Med. Sci. Supplement: 63-77, May 1973.

3.-Phillips, S.F.: Diarrhea: A Current View of the Pathophysiology. Progress in Gastroent. 63(3): 495-517, 1972.

4.-Phillips, S.F.: Fluid and Electrolyte Fluxes in the Gut. Hospital Practice 8(3): 137-146, 1973.

5.-Schultz, S.G. and Frizzell, R.A.: An Overview of Intestinal Absorptive and Secretory Processes| Gastroent. 63(1): 161-170, 1972.

6.-Hendrix, T.R. and Bayless, T.M.: Digestion: Intestinal Secretion. Annual Review of Phy. siol. 32: 139-164, 1970.
7.-Herbst, J.J., Sunshine, P. and Kretchmer, N.: Intestinal Malabsorption in Infancy and Childhood. Advances in Ped. 16: 11-64, 1969.

8.-Schenk, E.A., Samloff, I.M. and Klipstein, F. A.: Morphology of Small Bowel Biopsies. The Am. J. of Clin. Nutr. 21(9): 944-961, Sept. 1968.

9.-Shearman, D.J., Parkin, D.M. and McCleeland, D.BL.: The Demonstration and Function in the Gastrointestinal Tract. Gut 13: 438-499, 1972.

10.-Toner, P.G., Carr. K.E., Ferguson, A. and Mackay, C.: Scanning and Transmission Electron Microscopic Studies of Human Intestinal Mucosa. Gut 11: 471-481, 1970.

11.-Donatson, R.M.: Normal Bacterial Populations of the Intestine and Their Relation to Intestinal Function. New Eng. J. Med. 270 (18): 938-945, April 30, 1964.

12.-Donalson, R. M.: Normal Bacterial Populations of the Intestine and Their Relation to Intestinal Function. New Eng. J. Med. 270(19): 994-1001, May 7, 1964.

13.-Donalson, R. M.: Normal Bacterial Populations of the Intestine and Their Relation to Intestinal Function (Concluded). New Eng. J. Med. 270(20): 1050-1056, May 14, 1964.

14.--Gorbach, S. L.: Intestinal Microflora. Gastroent. 60(6): 1110-1129, June 1971.

15.-Dammin, G. J.: Pathogenesis of Acute Clinical Diarrheal Disease. Fed. Proceed., Gastro. Part I 24(1): 35-38, January-February 1965.

16.-Grady, G. F. and Keusch, G. T.: Pathogenesis of Bacterial Diarrheas (first of two parts). New Eng. J. Med. 285(15): 831-900, 1971.

17.-Eichenwald, H. F. and McCracken, G. H.: Acute Diarrheal Disease. Med. Clin. of N.A. 54(2): 443-454, March 1970.

18.-Walker, W. A. and Hong, R.: Immunology of the Gastrointestinal Tract: Part. I. J. of Pediat. 83(4): 517-530, October 1973.

19.-Walker, W. A. and Hong, R.: Immunology of the Gastrointestinal Tract: Part II. J. of Pediat. 83(5): 711-720, November 1973.

20.-Bellanti, J. A.: Role of Local Gamma-A-Immunoglobulins in Immunity. Am. J. Dis. Child. 115: 239-246, February, 1968. 
21.-Heremans, J. F., Crabbe, P. A. and Masson, P. L.: Biological Significance in Exocrine Gamma-A-Globulin. Acta Med. Scand. 179 (Suppl 445): 84-88, 1966.

22.-Tomasi, T. B.: Secretory Immunoglobulins. New Eng. J. Med. 287(10): 500-506, September $7,1972$.

23.-Mata, L. J., Urrutia, J. J., Albertazzi, C., Pellecer, $O$, and Arellano, E.: Influence of Recurrent Infections on Nutrition and Growth of Children in Guatemala. Am. J. Clin. Nutr. 25: 1267-1275, November 1972.

24.-Kent, J. H. and Lindebaum, J.: Correlation of Jejunal Function and Morphology in Patients with Acute and Chronic Diarrhea in East Pakistan. Gastroent. 52(6): 972-984, June 1967.

25.-Luckey, T. J.: Gnotobiology in Ecology. Arm. J. Clin. Nutr. 23(11): 1533-1540, November 1970.

26.-Soderhjelm, L.: Infant Feeding Hygiene in Sweden. Acta Pediatr. Scan. 61: 565-570, 1972.

27.-Soriano, H. y Macaya, J.: Sindrome Diarreico Agudo. En Texto de Pediatría. J. Meneghello, ed., Intermédica, Buenos Aires, Argentina, pp. 278-299, 1972.

28.-Meneghello, J. y Macaya, J.: Tendencia de la Mortalidad por Diarrea en el Lactante, en los últimos 20 años, y su Relación con Medidas de Terapéutica Individual y Colectiva. Pediatría 15: 465-478, 1972. (Santiago, Chile).

29.-Macaya, J., Soriano, H. y Duffau, G.: Algunas Consideraciones sobre Sindrome Diarreico Agudo del Lactante con Especial Consideración del Período Epidémico 1970-1971, en el Area Norte de Salud de Santiago. Pediatría 15(4): 506-512, Octubre-Diciembre 1972.

30.-Soriano, H., Macaya, J., Duffau, G. y Aguiló, C.: El Sindrome Diarreico Agudo con Deshidratación del Lactante como Problema Asistencial en la Unidad de Lactantes del Servicio de Pediatría del Hospital Roberto del Río, Area Norte de Salud, V Zona S.N.S. 1970. Pediatría 15(4): 506-512, Octubre-Diciembre 1972.

31.-Buttiaux, R.: Les Maladies Diarrheiques Transmissibles due Nourrisson et de l'Enfant. WHO Bulletin 21: 386-390, 1959.

32.-Gordon, J. E.: Diarrheal Disease of Early Childhood-Worldwide Scope of the Problem. Annals N.Y. Academy of Sci. 176: 9-15, January $7,1971$.
33.-Hardy, A. V.: Diarrhoeal Disease of Infants and Children. Mortality and Epidemiology. Bull. WId. Hith. Organ. 21: 309-319, 1959.

34.-Scrimshaw', N. S., Taylor, C. E. and Gordon, J. E.: Weanling Diarrhea-Asynergism of Infection and Nutrition. Interactions of Nutrition and Infection, World Health Organization, Geneva, 1968.

35.-Walker, A. C. and Harry, J. F.: A Survey of Diarrhoeal Disease in Malnourished Aboriginal Children. Med. J. of Australia 1: 904911, 1972.

36.-Joe, L. K., Rukmono, B., Oemijati, S., Sahab, K., Newell, K. W., Hway, S. T., and Talogo, $R$. W.: Diarrhea Among Infants in a Crowded Area of Dyakarta, Indonesia. Bull. Wld. Hith. Organ. 34: 197-210, 1966.

37.-Mata, L. J., Catalán, Marco, A., and Gordon, $J$. E.: Studies of Diarrheal Disease in Central America. Am. J. Trop. Med. and Hygiene 15(4) : 632-638, 1966.

38--James, W. P. T.: Effects of Protein-Calorie Malnutrition on Intestinal Absorption. Annals N.Y. Academy of Sci. 176: 244261, January 7, 1971.

39.-Van Zijl, W. J.: Studies on Diarrhoeal Diseases in Seven Countries by the WHO Diarrhoeal Diseases Advisory Team. BuIl. WId. HIth. Organ. 35: 249-261, 1966.

40.-Spencer, I. W. F. and Coster, M. E. E.: The Epidemiology of Gastroenteritis in Infancy: Part I. S.A. Med. J. 43(46): 1391-1397, November 15, 1969.

41.-Spencer, I. W. F. and Coster, M. E. E. The Epidemiology of Gastroenteritis in Infancy: Part II. S.A. Med. J. (43) (47) : 1438-1442, November 29, 1969.

42.-Spencer, I. W. F. and Coster, M. E. E.: The Epidemiology of Gastroenteritis in Infancy: Part III. S.A. Med. J. 43(48): 1466-1472, December 1969.

43.-Verhoestraete, L. J. and Puffer, R. R.: Diarrhoeal Disease with Special Reference to the Americas. Bull. Wld. Hlth. Organ. 19: 23-51, 1958.

44.-Pemberton, J.: Diarrhoeal Diseases-International Comparisons. Epidemiology Reports on Research and Teaching, Oxford U. Prcss, 1963. 
45.-Robinson, M.: Infant Morbidity and Mortality. The Lancet 1: 788-794, April 7, 1951.

46.- Rosenstein, B. J.: Salmonellosis in Infants and Children. J. of Pediat. 70(1): 1-7, January 1967.

47.-Gleason, N. N., Horwitz, M. S., Newton, L. H. and Moore, G. T.: A Stool Survey for Enteric Organisms in Aspen, Colorado. Am. J. Trop. Med. and Hygiene 19(3): 480-484, 1970.

48.-Gorbach, S. L.: The Toxigenic Diarrheas. Hosp. Practice 8(5): 103-110, May 1973.

49.-Hardy, A. V. and Watt, J.: Studies of the Acute Diarrheal Diseases. Publ. Hlth. Reports $60(3)$ : 57-66, January 19, 1945

50.-Cohen, J.: Morbidity from Intestinal Infections in Israel. Israel J. Med. Sci 8(7): 965 971, 1972.

51.-Cunnick, W. R., Eide, K. A. and Smith, N. J.: Digestive Disease as a National Problem. Gastroent. 51(2): 246-252, February 1968.

52.-Dean, A. G. and Jones, T. C.: Seasonal Gastroenteritis and Malabsorption at an American Military Base in the Philippines. Am. J. Epidem. 95(2): 111-127, 1972.

53.-Braun, O. H.: E. Coli Enteritis in Germany: Epidemiology and Recent Research. Annals N.Y. Academy of Sci. 176: 126-134, January 7, 1971.

54.-Bhat, P., Myers, R. M. and Feldman, R. A.: Significance of the Incidence of Shigella Infection as Indicated by Results of a Longitudinal Study. Japan. J. Med. Sci. Biol. 23: 237-242, 1970.

55.- Knight, R.: Diarrhoeal Illness Among Travellers. Nursing Times 69: 769-770, June 14, 1973.

56.-Moffet, H. L., Shulenberger, B. S. and Burkholder, B. S.: Epidemiology and Etiology of Severe Infantile Diarrhea. J. Pediat. 72 (1): 1-14, January, 1968.

57.-Soriano, H., Navarrete, M. and Nichols, B. L.: Diarrheal Syndrome Mortality in Childhood: Magnitude of the Problem Throughout the World. En preparación para publicación.

58.- Soriano, H., Navarrete, M. and Nichols, B.L.: Diarrheal Syndrome Mortality in Childhood:
Magnitude of the Problem in the Americas. En preparación para publicación.

59.-Kouranym, M., Vásquez, M. A. and Mata, L. J.: Prevalence of Pathogenic Enteric Bacteria in Children of 31 Panamanian Communities. Am. J. Trop. Med. and Hygiene 20(4) : 608-615, 1971.

60.-Grenier, B., Rolland, J. C., Bardie, F., Audurier, A., Salmon, D., Jamet, D.: Etude de l'Equilibre Bacteriologique des Selles des Nourrissons Role du Staphylocoque Dans l'Etiologie des Gastroenterites Infantiles. Arch. Franc. Ped. 29: 1083-1096, 1972.

61.-South, M. A.: Enteropathogenic Escherichia Coli Disease. New Developments and Perspectives. J. Pediatr. 79(1): 1-11, July 1971.

62.-Yow, M. D., Melnick, J. L., Blattner, R. J., Stephenson, W. B., Robinson, N. M., and Burkhardt, M. A.: The Association of Viruses and Bacteria with Infantile Diarnhea. Am. J. of Epidem. 92(1): 33-39, 1970.

63.-Cramblett, H. G., Asimi, P. and Haynes, R. E.: The Etiology of Infectious Diarrhea in Infancy, with Special References to Enteropathogenic E. Coli. Annals N.Y. Academy of Sci. 176: 80-92, 1971.

64.- Mata, L. J., Gangarosa, E. J., Cáceres, A., Perera, D. R. y Mejicanos, M. L.: Epidemia de Disentería Shiga en Centroamérica I. Investigaciones Etiológicas en Guatemala, 1969. Bol. Oficina San. Pan. 71: 93-107, Agosto 1971.

65.-De Olarte, D. G., Trujillo, H., Agudelo, N., Nelson, J. D., Haltalin, K. C.: Treatment of Diarrhea in Malnourished Infants and Children. Am. J. Dis. Child. 127: 379-388, March 1974.

66.-Nathan, V. I. and Baker, S. J.: An Epidemic of Tropical Sprue in Southern India I: Clinical Features. Annals of Trop. Med. and Parasit 72(1): 1-14, January 1968.

67.-Santiago-Borrero, P. J., Maldonado, N. and Horta, E.: Tropical Sprue in Children. Trop. Pediat. 76(3): 470-479, 1970.

68.- James, J. W.: Longitudinal Study of the Morbidity of Diarrheal and Respiratory Infections in Malnourished Children. Am. J. Clin. Nutr. 25: 690-694, July 1972. 
69.-Calderón, A., Soriano, H., Macaya, J. y Duffau, T. G.: Sindrome Diarreico Agudo con deshidratación - Tratamiento Inicial. Pediatría 15: 439-443, 1972. (Santiago, Chile)

70.-Suriano, H., Aguiló, C., Macaya, J., Duffan, G. y Calderón, A.: Sindrome Diarreico Agudo con Deshidratación del Laciante. Correlación Clínico-Humoral. Pediatría 15(4) : 425-438, Octubre-Diciembre 1972.

71.-Mata, L. J., Luttman, R. y Sánchez, L.: Micruorganismos Enteropatógenos en Niños con Diarrea Severa. Rev. Chil. Ped. 15(4): 176184, Diciembre 1964.

72.-Eiger, T. and D'Empaire, M.: Pathogenus Enterobacteria in Stool Cultures I. A Comparative Study. Acta Gastrosnt. Lat. Am. 4: 175180, 1972.

73.- Scardino, P. T.: Bacterila Diarrea in Eastern Nicaragua. S. Med. J. 64(7): 823-829, July 1971.

74.-Klipstein, F. A., Goldeman, L. V., Corcino, J. $J$. and Moore, W. E. C.: Enterotoxigenic Intestinal Bacteria in Tropical Sprue. Annals of Internal Med. 79: 632-641, 1973.

75.-Blacklow, N. R., Dolin, R., Fedson, D. S., Dutpont, H., Northrup, R. S., Hornick, R. B., and Chanock, R. M.: Acute Infectious Nonbacterial Gastroenteritis: Etiology and Pathogenesis. Annals of Internal Mied. 76(6): 993-1008, June 1972.

76.-Formal, S. B., Dupont, H. L., Hornick, R. B.: Enterotoxic Diarnheal Syndrome. Annual Revicw of Med. 24: 103-108, 1973.

77.-Banwell, J. G. and Sherr, H.: Effect of Bacterial Enterotoxins on the Gastrointestinal Tract. Prog. in Gastroent. 65(3)-467-497, September 1973.

78.-Bergogne-Berezin, E., Zechovsky, N., Cerf, M., Pappo, M.: Etude Critique de 100 Tubages Jejunaus. Essai de Confrontation des Donnees Cliniques et Bacteriologiques. Pathologie-Biologie 21: 505-514, May 1973. ('Paris).

79.-Bishop, R. F., Davidson, G. P., Holmes, I. H. and Ruck, B. J.: Virus Particles in Epithelial Cells of Duodenal Mucosa from Children with Acute Non-Bacterial Gastroenteritis. The Lancet 2: 1281-1283, December 8, 1973.

80-Bishop, R. F., Davidson, G. P., Holmes, I. H. and Ruck, B. J.: Detection of a New Virus by Electron Microscopy of Faecal Extracts from Children with Acute Gastroenteritis. The Lancet 1: 149-151, February 2, 1974.

81.-. Rodrigue, W. J, Lee Cline, W., larrot, R. H. and Chanock, R. M.: Reoviruslike Agent in Stools: Association with Infantile Diarrhea and Development of Serolgic Tests. Science 185: 1049-1053, 1974.

82.- Neal, R. A.: Progress Report, Pathogenesis of Amoebiasis. Gut 12: 483-486, 1971.

83.-Gyles, C. L.: Plasmids in Intestinal Bacteria. Am. J. Clin. Nutr. 25: 1455-1458, December 1972.

84.-Cohen, S.: Ecologic Conscquences of Resistance-Transfer Factors. Am. J. Clin. Nutr. 23(11): 1480-1487, November 1970.

85.-Dulaney, E., Lasken, L., and Allen, 1.: The Problems of Drug-Resistant Pathogenic Bacteria. Annals N.Y. Academy of Sci. 182: 5-415, June 1971.

86.- Kromery, V., Watanabe, L. and Roswal, T., eds.: Bacterial Plasmids and Antibiotic Resistance. Springer-Verlag, N.Y., 1972.

87.-Clowes, R. C.: Molecular Structure of Bacterial Plasmids. Bact. Rev. 36: 361-405, 1972.

88.-Meynell, G. G.: Bacterial Plasmids. M.I.T. Press, Cambridge, Mass., 1973.

89.-Davidson, G. P., Bishop, R. F., Townley, R. $R$. W., Holmes, I. H, and Ruck, B. J.: Importance of a New Virus in Acute Sporadic Enteritis in Children. The Lancet 1: 242-246, February 1, 1975.

90--Middleton, J. P., Szymamsky, T. M., Abbott, G. D., Bortolussi, R., and Hamilton, J. R.: Orbivirus Acute Gastroenteritis of Infancy. The Lancet Part 2, 1(7869): 1241-1244, June 22, 1974.

91.-Flewett, T. H., Bryden, A. S., Davies, H., Woode, G. N., Bridger, J. C., and Derrick, J. M.: Relation between Viruses from Acute Gastroenteritis of Children and Newborn CaIves. The Lancet 2: 61-63, July 13, 1974.

92.-Viteri, F. E., Flores, J. M., Alvarado, J., and Béhar, M.: Intestinal Malabsorption in Malnourished Children Before and During Recovery. Dig. Dis. 18(3): 201-211, 1973. 
93.-Kingston, M. E.: Biochemical Disturbances in Breast-Fed Infants with Gastroenteritis and Dehydratation. Trop. Ped. 82(6); 1073-1081, June 1973.

94.-Hazlewod, C. F., Nichols, B. L. and Fikes, W. R.: Theoretical Computation of Intracellular Ion Concentration. Proceed. Sympo. Bio med. Engin. 1: 397-398, 1966.

95.-Hazlewood, C. F. and Nichols, B. L.: An In Vitro Study of Resting Muscle Membrane Potential in Preweanling and Weanling Rats. Nature 213: 935, 1967.

96.- Hazlewood, C. F. and Nichols, B. L.: Skeletal Muscle Cell Polariation as a Function of Age. Intracellular Glass Microelectrodes Conference, University of Montreal, Montreal, Canada, in conjunction with Corning Glass Works, May 23-25, 1967.

97.- Asakura, H., Morita, T., Tsuchiya, M., Fukumi, H., Ohashi, M., Uylangco, C., and Castro, A.: Pathologic Findings from Intestinal Biopsy Specimen in Human Cholera. Dig. Dis. 18(4): 271-278, April 1973.

98.-Klipstein, F. A., Schenke, E. A.: Enterotoxigenic Intestinal Bacteria in Tropical Sprue, II. Effect of Bacteria and Their Enterotoxin on Intestinal Structure. Gastroent. 66: A-71/ $725,1974$.

99.-Keushc, G. T., Grady, G. F., Takeuchi, A. and Sprinz, H.: The Pathogenesis of Shigella Diarrhea II. Enterotoxin Induced Acute Enteritis in Rabbit Ileum. J. Inf. Dis. 126: $92-$ 95, 1972.

100.-Agus, S. G., Dolin, R., Wyatt, R., Tousimis, A. J. and Northrup, R. S.: Acute Infectious Non-bacterial Gastroenteritis: Intestinal Histopathology. Annals of Internal Med. 79: 18$25,1973$.

101.-Duffau, G., Macaya, J., Soriano, H. y Calderón, A.: Sindrome Diarreico Agudo con Deshidratación del Lactante. Hallazgos Necrópsicos. Pediatría 15: 459-464, Octubre-Diciembre 1972.

102.-Barnes, G. L. and Townley, R. R. W.: Duodenal Mucosal Damage in 31 Infants with Gastroenteritis. Archives of Disease in Childhood 48: 343-349, 1973.

103.-Toccalino, H., Licastro, R., Cardo, A. and Williams, M.: Histological Alterations of the Small Intestine in Normal Infants with Acute
Diarrhea. Acta Gastroen. Latinoamerica 4: 129-234, July-September 1972.

104.-Lifshitz, F., Wapnir, R. A., Wehman, H. C., Hawkins, R. L. and Pergolizzi, R.: Enteric Microbial Effects on Jejunal Carbohydrate Transport, Disacchardase Activity, Bile Salts and Ultrastructure. Gastro. 66: A-77/731, 1974.

105.-Lifshitz, F., Ramirez, P., Gutiérrez, G., and Cornado, M. C.: Carbohydrate Intolerance in Infants with Diarrhea. J. Ped. 79: 760-767, 1971.

106.-Torres-Pinedo, R., Rivera, C., and Rodriguez, $H .:$ Intestinal Absorptive Defects Associated with Enteric Infection in Infants. Annals of N.Y. Academy of Sci. 176: 284-298, January, 1, 1971.

107.-Ament, M. E.: Malabsorption Syndromes in Infancy and Childhood. Part I. J. Ped. 81 (4): 685-697, 1972.

108.-Poley, J. R.: Chronic Diarrhea in Infants and Children, Part I. S. Med. J. 66(9): 1035-1049, September, 1973.

109.-Poley, J. R.: Chronic Diarrhea in Infants and Children, Part II. S. Med. J. 66(10): 11331141, October, 1973.

110.-Poley, J. R.: Causes of Chronic Diarrhea in Infants and Children. Practical Ped. Postgraduate Med. 48: 143-147, December, 1970.

111.- Nelson, J.D. and Haltalin, K. C.: Accuracy of Diagnosis of Bacterial Diarrheal Disease by Clinical Features. J. Ped. 78(3): 519-522, March, 1971.

112.-Nichols, B. L., Hazlewood, C. F. and Barnes, $D$. J. Percutaneous Needle Biopsy of Quadriceps Muscle Electrolyte Analysis in Normal Pediatric Subjects. J. Ped. 72: 840-852, 1968.

113.-Harris, J. C., Dupont, H. L. and Hornick, R. $B .:$ Fecal Leukocytes in Diarrheal Illnes. Ann. Inter. Med. 76: 697-703, 1972.

114.-Soriano, H., Macaya, J., Duffau, G., García, I. y Urbina, A. N.: Sindrome Diarreico Agudo del Lactante. Empleo de la Reacción de Fehling en el Diagnóstico de la Intolerancia a los Disacáridos. Pediatría 15(4) : 452-458, OctubreDiciembre, 1972.

115.-Nichols, B. L., Alvarado, J. Rodriguez, J., Hazlewood, C. F. and Viteri, F. E.: Therapeutic 
Implications of Electrolyte, Water and Nitrogen Losses During Recovery from ProteinCalorie Malnutrition. J. Ped. 84(5): 759-768, 1974.

116.- Nichols, B. L., Alvarado, J., Hazlewood, C. F. and Viteri, F. E.: Clinical Significance of Muscle Potassium Depletion in Protein-CaIorie Malnutrition. J. Ped. 80(2) : 319-330, 1972.

117.-Aguilo, C., Soriano, H., Rizzardini, M. y Macaya, J.: Ampicilina en el Tratamiento de la Diarrea Aguda del Lactante. Pediatría 11: 263-271, 1968. (Santiago, Chile).

118.-Aguiló, C., Soriano, H., Guerrero, P. y Pino, C.: Acido Nalidixico en el Tratamiento de la Diarrea Aguda del Lactante. Pediatría 11: 224-231, 1968. (Santiago, Chile).

119.-Weissman, J. B., Gaugarosa, E. J., Dupont, $H$. L., Nelson, J. D. and Haltalin, K. C.: Shigellosis. To Treat or not to Tneat? JAMA 229(9): 1215-1216, 1974.

120.-Drachman, R. H.: Acute Infectious Gastroenteritis. Ped. Clin. of N.A. 21(3): 711-737, 1974.

121.-Dupont, H. L., Hornick, R. B.: Adverse Effect of Lomotil Therapy in Shigellosis. JAMA 226: 1525-1528, 1973.

122.-Tamer, M. A., Santora, $R$. and Sandberg, $D$. $H$.: Cholestyramine Therapy for Intractable Diarrhea. Pediatrics 53(2): 217-220, February 1974.

123.-Sherman, J. O., Hamly, C. A. and Khachadurian, $A . K$.: Use of an Oral Elemental Diet in Infants with Severe Intratable Diarrhea. J. Ped. 86(4): 518-523, 1975.

124.-Park, N., Bernier, L., Duffau, G., Macaya, J., Soriano, H., Muñoz, P., Hidalgo, R., Soto, A., Serené, N. y Tagle, M. A.: Fórmula de Garbanzo (Cicerarietinum) en el Tratamiento Dietético del Sindrome Diarreico Agudo. Pediatría 17: 71-74, 1974. (Santiago, Chile).

125.-Klish, W. J., Potts, E., Ferry, G. D. and Nichols, B. L.: Modular Formula: An Approach to Management of Infants with Complex Food Intolerances. Submitted for publication, J. Pediat.
126.-Dudrick, S. J., Copeland III, E. M. and Mac Fayden, Jr., B. V.: Long-Term Parenteral Nutrition. Its Current Status. Hosp. Practice 10(5): 47-58, May 1975.

127.-Shwachman, H., Lloyd-Still, J. D., Khaw, K. T. and Antonowicz, I.: Protracted Diarrhea of Infancy Treated by Intravenous Alimen. tation. Am. J. Dis. Child. 125: 365-368, March 1973.

128.-Bounous, G., Devroede, G., Haddad, H., Beaudry, R., Perey, B. J. and Lejeune, L. P.: Use of an Elemental Diet for Intestinal Disorders and for the Critically Ill. Dis. Col. and Rect. 17: 157-165, March-April, 1974.

129.-Andersson, H.: Treatment of Steatorrhea with Fat-Reduced Diet in Small Bowel Disease. Bibl. (Nutri. Diet.) 19: 6469, 1973.

130.-Ricour, C. and Nihoul-Fekete, C.: Nutrition Parenteral Prolongee Chez L'Enfant. Arch. Franc. Ped. 30: 469-490, 1973.

131.-Maheshkumar, A. P., Harberg, F. J., Nichols, $B$. L. and Brooks, B. F.: The Technique and Management of Parenteral Hyperalimentation in Children. Preliminary Report. St. Joseph's Med. Surg. J. 6: 97-103, 1971.

132.-Heird, W. C. and Winter, R. W. Total Intravenous Alimentation. Am. J. Dis. Child. 126: 287-289, 1973.

133.-Calderón, A., Goldenberg, E., Macaya, J., Bernier, L., Duffau, G., Emilfork, M., Soriano $H$. y Urbina, A. M.: Sindrome Diarreico de Evolución Tórpida. Análisis Clínico de $100 \mathrm{~Pa}-$ cientes. Pediatría 17: 95-102, 1974. (Santiago, Chile).

\section{AGRADECIMIENTOS}

Los autores agradecen a sus colegas de la Sección de Nutrición y Gastroenterologia del Departamento de Pediatría de la Escuela de Medicina de Baylor; a sus colegas de la Uniclad de Lactantes del Departamento de Pediatría del Hospital Roberto
del Rio y Facultad de Medicina de la Universidad de Chile, Area Norte; al Dr. Russell Blattner, Profesor y Jefe del Departamento de Pediatría de Baylor: y al Dr. Julio Meneghello, Profesor Jefe del Departamento de Pediatria del Hospital Roberto del Río. Sin la colaboración y apoyo de los arriba mencionados no habria sido posible preparar una actualidad como esta.

Los autores agradecen también a la Sra. Marilyn Navarretc su valiosa colaboración editorial a la preparación de este artículo.

Los autores desean expresar su reconocimicnto por la ayuda financiera para la preparación de este trabajo a las sizuientes instituciones: Instituto Nacional de Salud de EE. UU. (NIH) instituciones: Instituto Nacional de Salud de EE, UU. (NIH),
División de Recursos para la Investigación, Programa de Centros de Investigación Clínica General, fondo de ayuda RR-00188 administrado por el Centro de Investigación Clínica del Hospital de Niños de l'exas. Instituto Nacional de Salud de EE. UU., fondos de ayuda 29.05721 y GM 20154; y Administración Nacional Aeronáutica y Espacial (NASA), fondo de ayuda NGR-44-003-053. 